\title{
Postoperative complications of powered intracapsular tonsillectomy and monopolar electrocautery tonsillectomy in teens versus adults.
}

\author{
Douglas R Johnston \\ Thomas Jefferson University \\ Michael Gaslin \\ Thomas Jefferson University \\ Maurits Boon \\ Thomas Jefferson University \\ Edmund.Pribitkin \\ Ellow this and additional works at: https://jdc.jefferson.edu/otofp \\ Thomas Jefferson University \\ Part of the Otolaryngology Commons \\ pavid Rosenpowhingwivacess to this document benefits you
}

\section{Recommended Citation \\ Johnston, Douglas R; Gaslin, Michael; Boon, Maurits; Pribitkin, Edmund; and Rosen, David, "Postoperative complications of powered intracapsular tonsillectomy and monopolar electrocautery tonsillectomy in teens versus adults." (2010). Department of Otolaryngology - Head and Neck Surgery Faculty Papers. Paper 14. \\ https://jdc.jefferson.edu/otofp/14}

This Article is brought to you for free and open access by the Jefferson Digital Commons. The Jefferson Digital Commons is a service of Thomas Jefferson University's Center for Teaching and Learning (CTL). The Commons is a showcase for Jefferson books and journals, peer-reviewed scholarly publications, unique historical collections from the University archives, and teaching tools. The Jefferson Digital Commons allows researchers and interested readers anywhere in the world to learn about and keep up to date with Jefferson scholarship. This article has been accepted for inclusion in Department of Otolaryngology - Head and Neck Surgery Faculty Papers by an authorized administrator of the Jefferson Digital Commons. For more information, please contact: JeffersonDigitalCommons@jefferson.edu. 


\title{
As submitted to:
}

\section{The Annals of otology, rhinology, and laryngology.}

\author{
And later published as: \\ Postoperative complications of powered intrascapsular \\ tonsillectomy and monopolar electrocautery tonsillectomy in \\ teens versus adults.
}

\author{
July, 2010, Volume 119, Number 7, pp.485-9.
}

Johnston DR, Gaslin M, Boon M, Pribitkin E, Rosen D

Department of Otolaryngology - Head and Neck Surgery, Thomas Jefferson University Hospital, Philadelphia, PA

Correspondence:

David Rosen, MD

Thomas Jefferson University

Department of Otolaryngology - Head and Neck Surgery

925 Chestnut St., $6^{\text {th }}$ Floor

Philadelphia, PA 19130

David.Rosen@jefferson.edu 


\section{Abstract}

\section{Backgroud:}

Powered intracapsular tonsillectomy is a method for removing tonsils that leaves a small rim of tonsil tissue to protect the oropharynx, potentially resulting in less postoperative pain and a lower rate of postoperative bleeding. Although intracapsular tonsillectomy has been studied in children, there are no published reports about its use in the adult population.

\section{Objectives:}

1. Report the risks and benefits of intracapsular tonsillectomy when used in adults. 2. Report the complication rate of adult intracapsular tonsillectomy and compare this to traditional monopolar electrocautery tonsillectomy. 3. Demonstrate that adult intracapsular tonsillectomy is a safe and effective procedure for both chronic tonsillitis and adenotonsillar hypertrophy

\section{Methods:}

A retrospective chart review was performed over a 6 year period, from January 2000 to July 2006. All patients age 12 or over undergoing tonsillectomy for chronic tonsillitis or obstructive adenotonsillar hypertrophy were reviewed. Outcome measures included postoperative dehydration, postoperative hemorrhage, postoperative episodes of tonsillitis, and need for completion tonsillectomy.

\section{Results:}

A total of 589 patients were included in the study. Of those patients, 218 underwent intracapsular tonsillectomy and 361 underwent a traditional monopolar electrocautery tonsillectomy. The rate of postoperative hemorrhage requiring reoperation was significantly lower in the intracapsular tonsillectomy group, 1.8 versus $6.9 \%(\mathrm{p}=0.021)$. The rate of postoperative dehydration requiring readmission was also significantly lower in the intracapsular tonsillectomy group, $0.5 \%$ versus $3.1 \%(p=0.066)$. Recurrent pharyngitis after surgery occurred in $7.3 \%$ of intracapsular tonsillectomy patients and $2.8 \%$ of monopolar electrocautery tonsillectomy patients $(p=0.022)$. The rate of completion tonsillectomy was $1.32 \%$.

\section{Conclusions:}

Powered intracapsular tonsillectomy is an effective operation for adults with symptoms of chronic tonsillitis or adenotonsillar hypertrophy. It is associated with fewer short term complications of postoperative bleeding and dehydration than monopolar electrocautery tonsillectomy, but has a higher rate of recurrent pharyngitis with an acceptable rate of completion tonsillectomy. 


\section{Introduction}

Tonsillectomy has proven to be an effective procedure in adults for the treatment of chronic tonsillitis because it improves quality of life, healthcare dollar utilization, and overall economic burden (Bhattacharyya; Hsu; Mui; Fujihara; Bhattacharyya). The procedure of tonsillectomy has been modified many times over the years in terms of technique and instrumentation (Koempel). The studies comparing one technique to another have largely been conducted in the pediatric population. Powered intracapsular tonsillectomy (PIT) with a microdebrider has been compared in recent years to the monopolar electrocautery tonsillectomy (MET) in multiple studies (Schmidt; Schmidt 2; Solares; Sorin; Lister; Sobol; Derkay; Koltai). These two tonsillectomy techniques, however, have never been compared in the adult population. The purpose of our study was to retrospectively review our experience with PIT and MET in adults in order to compare the rate of reoperation for bleeding, the rate readmission for dehydration, and the postoperative incidence of tonsillitis, and need for completion tonsillectomy.

PIT has been shown in various studies in the pediatric population to be advantageous over traditional electrocautery tonsillectomy in several respects, including less postoperative pain (Lister; Derkay; Koltai), a lower incidence of delayed hemorrhage (Schmidt 2; Solares), a lower incidence of readmission for dehydration (Schmidt 2; Solares) less time until resumption of near-normal dietary intake (Derkay, Sobol), and quicker resumption of normal activity (Derkay). These advantages have not been universally demonstrable in all existing studies, but the general consensus at our institution is that the PIT is less painful because the pharyngeal musculature is not 
exposed or cauterized as in the MET. We believe this reduced pain equates to more rapidly resuming normal diet and work behaviors in adults.

The proposed disadvantages to the PIT are that the residual tonsillar tissue that remains in the fossae has a potential for reinfection and, possibly, regrowth, which may necessitate a completion tonsillectomy for either recurrent tonsillitis or tonsillar hypertrophy. Many of the existing studies have not had sufficient follow up to report on the incidence of postoperative tonsillitis in children undergoing tonsillectomy, except for the 2007 study by Schmidt, et al. Schmidt and colleagues retrospectively compared the postoperative tonsillitis rate in 49 PIT patients with 117 MET patients and found no statistical difference after a nearly two year follow up period. Sorin and colleagues found that 9 of $278(3.2 \%)$ pediatric PIT patients had tonsillar regrowth, two of whom required reoperation for completion tonsillectomy to definitively manage obstructive sleep apnea (Sorin).

The main objective of our study was to determine if PIT was a safe and effective procedure in adults for the treatment of both chronic tonsillitis and adenotonsillar hypertrophy. From a technical perspective, tonsillectomy in adults can differ from tonsillectomy in children because adults have larger blood vessels and may have a greater degree of tonsillar fibrosis and intraoperative bleeding from chronic tonsillitis. Our main outcome measures were to determine if PIT resulted in similar rates of reoperation for postoperative hemorrhage and rates of readmission for dehydration. Additionally, we 
sought to establish if the postoperative rates of tonsillitis and reoperation for completion tonsillectomy were similar between the PIT and MET groups.

\section{Methods}

All research was performed after obtaining approval from the institutional review board at the participating hospital. All surgeries were performed by one of three attending surgeons with the assistance of residents.

A retrospective chart review was conducted for all adult patients. Adult patients were defined as 12 or greater for tonsillectomy because this is the age cutoff in the medicare billing definition of adult tonsillectomy. The adult patients undergoing primary tonsillectomy over a 6-year period, from January 2000 to July 2006 were identified via billing codes. To be included in the study, the patients were required to be at least 12 years of age at the time of tonsillectomy, have undergone either a PIT or MET with or without adenoidectomy. Patients were excluded from analysis if they had undergone simultaneous procedures in the upper aerodigestive tract (functional endoscopic sinus surgery, septoplasty, turbinate reduction, uvulopalatopharyngoplasty) or if they had less than two months of follow up. The hospital electronic medical records and office charts of patients meeting the inclusion criteria were reviewed and data collected for age, sex, indication for tonsillectomy, procedure performed, operative time, estimated blood loss during the procedure, and any office visit, emergency room visit, hospital admission, or operation performed after the procedure. 
The primary outcomes of the study were severe bleeding requiring reoperation and severe dehydration requiring admission. Bleeding complications were divided into minor bleeding (patient was seen for bleeding but not admitted), intermediate bleeding (patient was admitted for observation but no operation performed to control hemorrhage), and severe bleeding (requiring reoperation). Complications for dehydration were likewise separated into minor (requiring a visit to the office or emergency room without admission) or major (requiring admission). Long term complications of recurrent pharyngitis or tonsillitis were determined by examining the patient's office chart and emergency room records. Any visit at least four weeks postoperatively with a primary diagnosis of pharyngitis or tonsillitis was considered to be a recurrence.

Chi Square analysis was used to determine significance of differences in age, sex, and indication for operation preoperatively. Fisher's Exact Test was used to determine the significance of the differences in the primary outcomes of bleeding and dehydration rates, as well as the secondary outcomes of recurrent tonsillitis and completion tonsillectomy rates. The Wilcoxon Signed-Rank Test was used to determine significance of differences between operative times and blood loss between the two procedures.

We also assessed the association between procedure type, indication for the tonsillectomy, patient sex, and patient age with the outcomes of major hemorrhage requiring reoperation and major dehydration requiring readmission. Because of the small number of events for these two outcomes, exact analytic methods were used. Univariable analyses were based on Fisher's exact test. Multivariable analyses to assess the 
independent effect of each predictor variable on each of the two outcomes were based on exact conditional logistic regression. Odds ratios, $95 \%$ confidence intervals, and p-values were computed. Statistical analyses were conducted in LogXact 8 (Cytel Inc., Cambridge, MA).

\section{Results}

A total of 743 patients were identified who met the inclusion criteria for entry into the study. Of these, 164 patients had simultaneous upper aerodigestive tract procedures and were excluded, leaving 579 patients for analysis. All included patients had a minimum of two months follow up, with a median follow up of 32 months.

The demographics of the two groups of patients are summarized in Table 1. There were no significant gender differences between the two groups ( $\mathrm{p}=0.39$ ), although approximately twice as many women underwent tonsillectomy as men (66\% vs. $34 \%)$. There was a significant difference $(\mathrm{p}<0.0002)$ in age between the two groups, with the intracapsular tonsillectomy group being younger by an average of 4 years (23.1 vs. 27.1). There were proportionately more patients with chronic tonsillitis in the traditional tonsillectomy group (77.3\% vs. $66.5 \%)$, although this did not reach statistical significance $(\mathrm{p}=0.15)$.

The primary outcomes of this study are severe bleeding requiring reoperation and severe dehydration requiring admission. 
Hemorrhage. There were a total of 44 (7.6\%) cases of hemorrhage, including 6 mild, 9 intermediate, and $29(5.0 \%)$ cases of major hemorrhage that required reoperation. The reoperation rate for major hemorrhage by procedure type, indication, sex, age, and operating surgeon is summarized in Table 2. In the multivariable model that assessed the effect of these four variables simultaneously, the risk of major hemorrhage for PIT was estimated to be about one quarter that of MET (1.8\% vs 6.9\%) (odds ratio, $\mathrm{OR}=0.28, \mathrm{p}$ $=0.021)$. In addition, compared to females, males had almost a threefold higher risk of major hemorrhage $(\mathrm{OR}=2.87, \mathrm{p}=0.011)$. Indication and age were not significantly associated with major hemorrhage.

These results did not change when operating surgeon was added to the model. Procedure type and sex remained significant $(\mathrm{OR}=0.28, \mathrm{p}=0.026$, for the former; $\mathrm{OR}=3.02, \mathrm{p}=$ 0.006 , for the latter), while there was no significant difference of major hemorrhage rates across the three surgeons $(\mathrm{p}=0.589)$.

When all hemorrhages were considered (as opposed to only major hemorrhages), the effect of procedure type was attenuated $(\mathrm{OR}=0.53, \mathrm{p}=0.124)$, while sex remained significant $(\mathrm{OR}=2.19, \mathrm{p}=0.016)$. Neither indication nor age was significant $(\mathrm{p}=0.193$ and 0.145 , respectively).

Dehydration. A total of $16(2.8 \%)$ cases of dehydration were noted, including $12(2.1 \%)$ cases of major dehydration requiring readmission. The readmission rate by procedure type, indication, sex, age, and operating surgeon is summarized in Table 3. In the 
multivariable model, PIT had less than one sixth the risk of major dehydration compared to $\operatorname{MET}(0.5 \%$ vs. $3.1 \%)(\mathrm{OR}=0.17, \mathrm{p}=0.066)$. In contrast to hemorrhage, females appeared to be in much higher risk of dehydration than males, with all twelve cases of readmission for dehydration being females. In the multivariable model, the risk of major dehydration among males was estimated to be about one seventh that among females (OR $=0.14, p=0.022$ ). Indication and age were not significantly associated with major dehydration.

When operating surgeon was added to the model, the effects of procedure type and sex remained largely unchanged, although the former became non-significant $(\mathrm{OR}=0.21, \mathrm{p}=$ $0.171)$, while the latter remained significant $(\mathrm{OR}=0.13, \mathrm{p}=0.019)$. There was no significant difference of major dehydration rates across the three surgeons $(\mathrm{p}=0.486)$.

The long term complication of recurrent tonsillitis or pharyngitis is significantly greater in patients who underwent PIT $(7.3 \%$ vs. $2.8 \%, \mathrm{p}=0.022)$. The rate of reoperation for PIT was $1.3 \%$. Table 5 summarizes the four patients who required a completion tonsillectomy after PIT.

Intraoperative times were similar between the two groups, with PIT taking on average 4.4 minutes longer (35.4 vs. 31.0 minutes, $\mathrm{p}=0.071)$. Intraoperative blood loss was higher in the PIT group (110 vs. 83 milliliters, $\mathrm{p}=0.00018)$. These data are presented in Table 5.

\section{Discussion}


This study is the first in the literature comparing PIT to MET in the adult population. Our results demonstrate that PIT is a safe alternative to MET with decreased rates of reoperation for bleeding $(1.8 \%$ vs. $6.9 \%, \mathrm{p}=0.021)$ and readmission for dehydration $(0.5 \%$ vs. $3.1 \%, \mathrm{p}=0.066)$. We also found that patients undergoing PIT were more likely to have recurrent tonsillitis $(7.3 \%$ vs. $2.8 \%, \mathrm{p}=0.022)$ and a small percentage may require completion tonsillectomy $(1.3 \%)$.

With regard to postoperative readmission for dehydration, it was clear that PIT results in far fewer readmissions $(0.5 \%$ vs. $3.1 \%, \mathrm{OR}=0.17, \mathrm{p}=0.066)$. It is our belief that the reduced postoperative pain in the PIT group due to less pharyngeal muscle irritation and inflammation results in a greater ability of these patients to maintain hydration and oral intake. Interestingly, women were statistically more likely to be readmitted for dehydration than men, regardless of the tonsillectomy technique $(\mathrm{OR}=0.14, \mathrm{p}=0.022)$. The literature does not describe a consistent gender predilection for increased pain or dehydration following tonsillectomy and are uncertain of the etiology. Although our study was not designed to assess postoperative pain, we believe PIT results in less postoperative pain compared to MET, which has been confirmed in a number of studies in the pediatric population (Lister; Derkay; Koltai). Our rate of readmission in the PIT group of $0.5 \%$ for dehydration secondary to pain also compares favorably to the results published by Schmidt, et al. and Solares, et al (Schmidt 2; Solares), although Schmidt and colleagues did not enumerate which patients were seen in the emergency room and treated versus those who were admitted for treatment. 
PIT represents an effective treatment option for chronic tonsillitis and tonsillar hypertrophy in adults that may provide a quicker return to normal activity and work. A more rapid return to work would mean less of an economic burden to society, although this must be factored against the long term costs of persistent tonsillitis or tonsillar regrowth, factors which are not addressed in this study. Additionally, a lower rate of reoperation for postoperative hemorrhage and lower rate of readmission for postoperative dehydration may also signify a cost savings, although this must be factored against an increased rate of completion tonsillectomy for tonsillar regrowth.

Our rate of reoperation for postoperative hemorrhage in the PIT group is slightly higher than the reported PIT rates under 1\% in children (Solares; Sorin; Koltai, Schmidt 2), which may be secondary to the larger surface area of the tonsillar fossae and larger vessels in adults. Similarly, our rate of reoperation for bleeding (severe bleeding) of $6.9 \%$ in the MET group is higher than the usual reported rates for post-tonsillectomy hemorrhage and reoperation of $2-4 \%$. Consistent with the published literature, the majority of reoperations in our series were the result of delayed hemorrhage (5-10 days) (Figure 2). This higher rate of reoperation for bleeding may be partly due to our departmental policy to generally operate rather than observe patients who are admitted for post-tonsillectomy hemorrhage. The statistically significant difference in bleeding rates between males and females, regardless of technique or indication has also been described in numerous studies, although the reason is unknown (Windfuhr, Kristenson, Roberts, Colclasure, Tomkinson). 
The long term complication of recurrent tonsillitis or pharyngitis was significantly greater in our patients who underwent PIT compared to MET ( $7.33 \%$ vs. $2.77 \%, \mathrm{p}=0.022)$. This recurrence is likely related to the small amount of retained tonsillar tissue. Schmidt and colleagues found that 49 pediatric patients who underwent PIT had no greater incidence of tonsillitis in comparison to the 117 who underwent MET in almost a two-year follow up period ( 0.26 vs. 0.17 infections per year of follow up, $\mathrm{p}=0.441)$ (Schmidt 1$)$. In their PIT group $16.3 \%$ of patients experienced tonsillitis in 21 months of follow up. The MET group had an incidence of tonsillitis of $14.5 \%$ in 24 months of follow up. Our rates of postoperative tonsillitis compare favorably at less than half of their reported rates with a median follow up of 32 months. In our judgement, the decreased risk of postoperative hemorrhage and decreased risk of readmission for dehydration outweighs the increased incidence of recurrent tonsillitis associated with PIT in the adult population.

Differences in intraoperative parameters for PIT and MET were also noted in this study. The duration of PIT was on average 4.4 minutes longer ( 35.4 vs. 31.0 minutes, $\mathrm{p}=0.071$ ). Although not clinically significant, this 4.4 minute time loss may translate into significant costs, depending on the volume of tonsillectomies an institution performs . Intraoperative blood loss was also higher in the intracapsular tonsillectomy group (110 vs. 83 milliliters, $\mathrm{p}=0.00018$ ), a finding of minimal clinical significance in this age population.

The results of our study should be examined with a few limitations in mind. The study has inherent weakness in that it is a retrospective study with several attending surgeons 
and resident surgeons. Additionally, the study has a degree of selection bias that favored PIT for younger patients (23.1 vs. 27.1 years old, $\mathrm{p}<0.0002)$, making a true comparison of the techniques impossible. There were also proportionately more patients with chronic tonsillitis in the traditional tonsillectomy group (77.3\% vs. $66.5 \%)$, although this did not reach statistical significance $(\mathrm{p}=0.15)$. Nonetheless, the large numbers of patients studied and relatively long median follow up of 32 months support the study's findings.

\section{Conclusions}

This large, retrospective single institution study demonstrates that PIT is a safe and efficacious procedure in adults. PIT results in an improved rate of post-tonsillectomy bleeding and readmission for dehydration compared to MET, regardless of the indication. PIT does have a higher, but acceptable rate of postoperative tonsillitis compared to MET and may require a completion tonsillectomy in only a small fraction of patients. 


\section{References}

Bhattacharyya N, Kepnes LJ, and Shapiro J: Efficacy and quality-of-life impact of adult tonsillectomy. Archives of Otolaryngology Head and Neck Surgery, 2001; 127(11): 1347-50.

Hsu AP, Tan KL, Tan YB, et al: Benefits and efficacy of tonsillectomy for recurrent tonsillitis in adults. Acta Otolaryngologica, 2007; 127(1): 62-64.

Mui S, Rasgon BM, and Hilsinger RL Jr.: Efficacy of tonsillectomy for recurrent throat infection in adults. Laryngoscope, 1998; 108(9):1325-28.

Fujihara K, Koltai PJ, Hayashi M, et al: Cost-effectiveness of tonsillectomy for recurrent acute tonsillitis. Annals of Otology, Rhinology, and Laryngology, 2006;115(5): 365-69. Bhattacharyya N, and Kepnes LJ: Economic benefit of tonsillectomy in adults with chronic tonsillitis. Annals of Otology, Rhinology, and Laryngology, 2002. 111(11): 98388.

Koempel, JA, Solares CA, and Koltai PJ: The evolution of tonsil surgery and rethinking the surgical approach to obstructive sleep-disordered breathing in children. Journal of Laryngology and Otology, 2006; 120(12): 993-1000.

Schmidt R, Herzog A, and Cook S, et al.: Powered intracapsular tonsillectomy in the management of recurrent tonsillitis. Otolaryngology Head and Neck Surgery, 2007; 137(2):338-40.

Schmidt R, Herzog A, and Cook S, et al: Complications of tonsillectomy: a comparison of techniques. Archives of Otolaryngology Head and Neck Surgery, 2007; 133(9): 92528. 
Solares CA, Koempbel JA, Hirose K, et al.: Safety and efficacy of powered intracapsular tonsillectomy in children: a multi-center retrospective case series. International Journal of Pediatric Otolaryngology, 2005; 69(1): 21-26.

Sorin A, Bent JP, April MM et. al: Complications of microdebrider-assisted powered intracapsular tonsillectomy and adenoidectomy. Laryngoscope, 2004; 114(2): 297-300. Lister MT, Cunningham MJ, Benjamin B, et al: Microdebrider tonsilltomy vs electrosurgical tonsillectomy: a randomized, double-blind, paired control study of postoperative pain. Archives of Otolaryngology Head and Neck Surgery, 2006. 132(6): 599-604.

Sobol SE, Wetmore RF, Marsh RR, et al: Postoperative recovery after microdebrider intracapsular or monopolar electrocautery tonsillectomy: a prospective, randomized, single-blinded study. Archives of otolaryngology head and neck surgery, 2006; 132(3): $270-74$.

Derkay CS, Darrow DH, Welch C, et al: Post-tonsillectomy morbidity and quality of life in pediatric patients with obstructive tonsils and adenoid: microdebrider vs electrocautery. Otolaryngology Head and Neck Surgery, 2006; 134(1): 114-20. Koltai PJ, Solares CA, Mascha EJ, et al: Intracapsular partial tonsillectomy for tonsillar hypertrophy in children. Laryngoscope, 2002; 112(8 Pt 2 Suppl 100): 17-19.

Windfuhr JP, Chen YS, and Remmert S: Hemorrhage following tonsillectomy and adenoidectomy in 15,218 patients. Otolaryngology - Head and Neck Surgery, 2005; 132(2): 281-86.

Kristensen S and Tveteras K: Post-tonsillectomy hemorrhage: a retrospective study of 1,150 operations. Clinical Otolaryngology, 1992; 17: 13-17. 
Colclasure JB and Graham SS: Complications of outpatient tonsillectomy and adenoidectomy: a review of 3,340 cases. Ear, Nose, and Throat Journal, 1990; 69: 15560.

Tomkinson A, DeMartin S, Gilchrist CR, et al: Instrumentation and patient characteristics that influence postoperative hemorrhage rates following tonsil and adenoid surgery. Clinical Otolaryngology, 2005; 30: 338-46. 
Table 1: Patient Demographics

\begin{tabular}{l|lccc}
\hline & & \multicolumn{1}{c}{ Intracapsular } & Traditional & p-value \\
\hline Sex & Male & $79(36.2 \%)$ & $117(32.4 \%)$ & 0.39 \\
& Female & $139(63.8 \%)$ & $244(67.6 \%)$ & \\
\hline \multirow{2}{*}{ Age } & Mean Age & 23.1 & 27.1 & $<0.0002$ \\
& Age Range & $12-56$ & $12-62$ & \\
\hline \multirow{2}{*}{ Indication } & Hypertrophy/Obstruction & $27(12.4 \%)$ & $25(6.9 \%)$ & \\
& Chronic Infection & $145(66.5 \%)$ & $279(77.3 \%)$ & 0.15 \\
& Both & $46(21.1 \%)$ & $57(15.8 \%)$ & \\
\hline \multicolumn{7}{l}{ Total patients } & 218 & 361 & \\
\hline
\end{tabular}

Table 2. Major hemorrhage requiring reoperation.

\begin{tabular}{|c|c|c|c|c|c|c|}
\hline & \multicolumn{3}{|c|}{ Univariable } & \multicolumn{3}{|c|}{ Multivariable } \\
\hline & $n$ & $(\%)$ & $P$ & $O R$ & $(95 \% C I)$ & $P$ \\
\hline Procedure & & & 0.006 & & & 0.021 \\
\hline Traditional bovie tonsillectomy & 25 & (6.9) & & 1.00 & Ref & \\
\hline Intracapsular tonsillectomy & 4 & $(1.8)$ & & 0.28 & $(0.07,0.85)$ & \\
\hline Indication & & & 0.624 & & & 0.750 \\
\hline Chronic infection & 24 & (5.7) & & 1.00 & Ref & \\
\hline Hypertrophy/Obstruction & 2 & (3.9) & & 0.59 & $(0.06,2.67)$ & \\
\hline Both & 3 & $(2.9)$ & & 0.64 & $(0.12,2.23)$ & \\
\hline Sex & & & 0.016 & & & 0.011 \\
\hline Female & 13 & (3.4) & & 1.00 & Ref & \\
\hline Male & 16 & $(8.2)$ & & 2.87 & $(1.24,6.79)$ & \\
\hline Age & & & 0.407 & & & 0.576 \\
\hline $12-20$ & 7 & $(3.2)$ & & 1.00 & Ref & \\
\hline $21-30$ & 13 & $(6.5)$ & & 1.93 & $(0.68,6.00)$ & \\
\hline $31-40$ & 6 & $(5.5)$ & & 1.45 & $(0.38,5.37)$ & \\
\hline$>40$ & 3 & (5.9) & & 1.67 & $(0.26,8.01)$ & \\
\hline Surgeon & & & 0.819 & & & \\
\hline DR & 15 & (5.2) & & & & \\
\hline EP & 8 & (4.3) & & & & \\
\hline MB & 6 & (5.8) & & & & \\
\hline
\end{tabular}

OR: odds ratio; 95\% CI: 95\% confidence interval; P: p-value.

Table 3. Major dehydration requiring readmission.

\begin{tabular}{|c|c|c|c|c|c|}
\hline & \multicolumn{2}{|c|}{ Univariable } & \multicolumn{3}{|c|}{ Multivariable } \\
\hline & $n \quad(\%)$ & $P$ & OR & $(95 \% C I)$ & $P$ \\
\hline $\begin{array}{l}\text { Procedure } \\
\text { Traditional bovie tonsillectomv }\end{array}$ & & 0.036 & & $D_{0}$ & 0.066 \\
\hline
\end{tabular}


Intracapsular tonsillectomy

\section{Indication}

Chronic infection

Hypertrophy/Obstruction

Both

Sex

Female

Male

$$
\begin{aligned}
& \text { Age } \\
& 12-20 \\
& 21-30 \\
& 31-40 \\
& >40
\end{aligned}
$$

\section{Surgeon}

DR

EP

MB
$1 \quad(0.5)$

0.790

$10 \quad(2.4)$

$0 \quad-$

2 (1.9)

$12(3.1)$

$0 \quad-$

$2(0.9)$

$8 \quad(4.0)$

$1 \quad(0.9)$

$1 \quad(2.0)$

$8 \quad(2.8)$

$1 \quad(0.5)$

3 (2.9)

0.011

0.120

0.158
$0.17 \quad(0.01,1.22)$

1.000

$1.00 \quad$ Ref

$1.28 \quad(0.00,9.07)$

$0.85 \quad(0.09,4.23)$

0.022

1.00 Ref

$0.14(0.00,0.88)$

0.344

$1.00 \quad$ Ref

$3.26 \quad(0.63,32.3)$

\begin{tabular}{|c|c|c|c|c|c|}
\hline Age & Sex & Procedure & Indication & Complication & $\begin{array}{l}\text { Timing of comp } \\
\text { tonsillectomy }\end{array}$ \\
\hline 37 & $\mathbf{M}$ & $\begin{array}{l}\text { Intracapsular } \\
\text { tonsillectomy }\end{array}$ & Chronic tonsillisits & $\begin{array}{l}\text { Bleeding, bilateral } \\
\text { peritonsillar abscess }\end{array}$ & 2 days postoper \\
\hline 17 & $\mathbf{F}$ & $\begin{array}{l}\text { Intracapsular } \\
\text { tonsillectomy }\end{array}$ & Chronic tonsillitis & Persistent tonsillitis & 6 months posto \\
\hline 19 & $\mathbf{M}$ & $\begin{array}{l}\text { Intracapsular } \\
\text { tonsillectomy }\end{array}$ & $\begin{array}{l}\text { Chronic tonsillitis and } \\
\text { tonsillar hypertophy }\end{array}$ & Persistent tonsillitis & 9 months posto \\
\hline 34 & $\mathbf{F}$ & $\begin{array}{l}\text { Intracapsular } \\
\text { tonsillectomy }\end{array}$ & Chronic tonsillitis & Persistent tonsillitis & 3 months posto \\
\hline
\end{tabular}

$0.79 \quad(0.02,15.5)$

$1.57 \quad(0.03,31.4)$

OR: odds ratio; 95\% CI: 95\% confidence interval; P: p-value.

Table 4: Patients Requiring Completion Tonsillectomy

Table 5: Intraoperative data

\begin{tabular}{l|cccc}
\hline & Value & Intracapsular & Traditional & p-value \\
\hline Estimated blood loss $(\mathbf{m l})$ & Mean & 110 & 83 & \multirow{2}{*}{0.00018} \\
& Range & $30-300$ & $30-250$ & \\
\hline Operative time (minutes) & Mean & 35.4 & 31.0 & \multirow{2}{*}{0.071} \\
& Range & $15-90$ & $13-87$ & \\
\hline
\end{tabular}

